\title{
Automatic Intra-operative Generation of Geometric Left Atrium/Pulmonary Vein Models from Rotational X-Ray Angiography
}

\author{
Carsten Meyer ${ }^{1}$, Robert Manzke ${ }^{2}$, Jochen Peters ${ }^{1}$, Olivier Ecabert ${ }^{1}$, \\ Reinhard Kneser ${ }^{1}$, Vivek Y. Reddy ${ }^{3}$, Raymond C. Chan ${ }^{2}$, and Jürgen Weese ${ }^{1}$ \\ ${ }^{1}$ Philips Research Laboratories, X-Ray Imaging Systems, \\ Weisshausstrasse 2, D-52066 Aachen, Germany \\ ${ }^{2}$ Philips Research North America, Clinical Sites Research, \\ 345 Scarborough Road, Briarcliff Manor, NY, USA \\ ${ }^{3}$ Experimental Electrophysiology Laboratory, Cardiac Arrhythmia Service, \\ Massachusetts General Hospital, Boston, USA \\ \{carsten.meyer, robert.manzke, juergen.weese\}@philips.com
}

\begin{abstract}
Pre-procedural imaging with cardiac CT or MR has become popular for guiding complex electrophysiology procedures such as those used for atrial fibrillation ablation therapy. Electroanatomical mapping and ablation within the left atrium and pulmonary veins (LAPV) is facilitated using such data, however the pre-procedural anatomy can be quite different from that at the time of intervention. Recently, a method for intra-procedural LAPV imaging has been developed based on contrastenhanced 3-D rotational X-ray angiography (3-D RA). These intraprocedural data now create a compelling need for rapid and automated extraction of the LAPV geometry for catheter guidance. We present a new approach to automatic intra-procedural generation of LAPV surfaces from 3-D RA volumes. Using model-based segmentation, our technique is robust to imaging noise and artifacts typical of 3-D RA imaging, strongly minimizes the user interaction time required for segmentation, and eliminates inter-subject variability. Our findings in 33 patients indicate that intra-procedural LAPV surface models accurately represent the anatomy at the time of intervention and are comparable to preprocedural models derived from CTA or MRA.
\end{abstract}

\section{Introduction}

Fluoroscopic X-ray guidance has long been the clinical standard for image-based guidance in interventional cardiac electrophysiology (EP) procedures. Recently, electroanatomical mapping (EAM) for deriving 3-D structural information during EP procedures has been demonstrated to improve the efficacy of catheter ablation 122. EAM has been further enhanced by the use of segmented surface models representing the left atrium and pulmonary veins (LAPV) imaged with volumetric techniques (MR/CT) 34. Left atrial geometry segmentation from these types of data is a well-known problem and has been an active area

D. Metaxas et al. (Eds.): MICCAI 2008, Part II, LNCS 5242, pp. 6169, 2008.

(C) Springer-Verlag Berlin Heidelberg 2008 
of research. Algorithms for LAPV segmentation have been presented for CT [5]6, CT-Angiography (CTA) 7] and MR-Angiography (MRA) 78]. During intervention, these models can be displayed with EAM or fluoroscopic data for catheter navigation. A distinct drawback is the rare availability of CT or MR in the intervention room. When using pre-procedural CT or MR images instead, LAPV anatomy may vary considerably between imaging and intervention [9]. To address these issues, intra-procedural volume imaging for interrogating cardiac anatomy in 3-D has been developed and validated, using contrast-enhanced 3-D rotational X-ray angiography (3-D RA) 91011 for near real-time imaging that integrates seamlessly into the current EP workflow.

Since intra-procedural volume imaging is now possible, a rapid, robust method for LAPV segmentation is essential to extract clinically-relevant surface anatomy with minimal interaction. This problem is challenging due to the quality of typical 3-D RA images. Artifacts include noise, catheter streak artifacts, indistinct chamber boundaries, and variable contrast density due to inhomogeneous mixing. The small number of acquired projection angles, low temporal resolution (as compared to $\mathrm{CT} / \mathrm{MR}$ ), low X-ray dose and presence of highly-attenuating catheters and electrode wires all degrade image quality. So far, surface extraction has been accomplished based on techniques which necessitate significant user interaction [10].

In this work, we present - to the best of our knowledge — the first algorithm for fully-automated, intra-procedural estimation of LAPV geometry from 3-D RA data. We compare the 3-D RA models to surfaces from pre-procedural CTA or MRA models, demonstrating results from 33 subjects.

\section{Methods and Materials}

\subsection{Intra-procedural Rotational X-Ray Imaging}

Intra-procedural imaging of the LAPV with 3-D RA has been described in 910]. Data acquisition was performed on 33 patients with the Philips Allura XPer FD10 flat detector single-plane X-ray system. Depending on heart size, single or dual rotational image acquisition was performed with a breath-hold using a $220^{\circ}$ rotation over $4 \mathrm{~s}$ at a sampling rate of 30 frames/s. Reconstruction was performed using the standard approximate Feldkamp (FDK) cone-beam algorithm. A grid size of $256^{3}$ with isotropic voxel dimension of $0.43 \mathrm{~mm}$ was used. In the case of dual acquisitions, the image volumes were fused before analysis [10. Examples of 3-D RA slices are presented in Fig. 1, in comparison to corresponding slices from pre-procedural CTA or MRA data. Fig. 1 clearly shows that the 3-D RA images suffer from lower image contrast, higher noise and image artifacts than the CTA or MRA data. The limited field-of-view leads to poor definition of the mitral annulus compared to CTA or MRA where it can be defined by surrounding heart structures. Therefore, automatic segmentation of LAPV anatomy from 3-D RA data is much more challenging than for CTA or MRA. 


\section{3-D RA}

(patient 23)

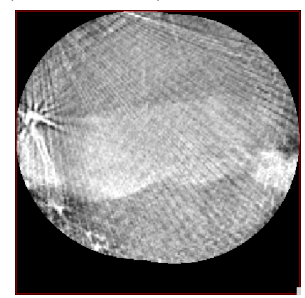

CTA (cut-out) (patient 23)

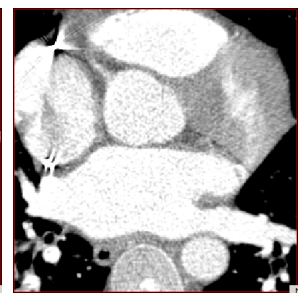

3-D RA

(patient 19)

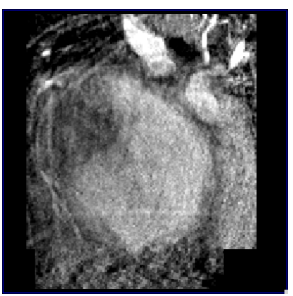

MRA (cut-out)

(patient 19)

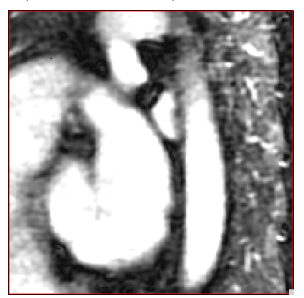

Fig. 1. Comparison of intra-procedural rotational X-ray angiographic images with preoperative CTA and MRA data. From left to right: 3-D RA short axis slice of patient 23, enlarged cut-out of 3-D CTA short axis slice of patient 23, 3-D RA sagittal slice of patient 19, enlarged cut-out of MRA sagittal slice of patient 19.

\subsection{Pre-procedural CTA/MRA}

Multi-slice cardiac CTA imaging was performed with a 64-slice scanner in 20 of 33 patients. Imaging parameters included: $120 \mathrm{kV}, 850 \mathrm{mAs}, 32 \times 0.625 \mathrm{~mm}$ collimation (sampled twice), and 0.33 s rotation time. ECG-gated reconstruction was performed on a $512 \times 512$ pixel matrix $(0.4-0.6 \mathrm{~mm}$ slice thickness, $20 \mathrm{~cm}$ fieldof-view). The remaining 13 patients underwent non-gated T1-weighted cardiac $\mathrm{MR}$ imaging $(\mathrm{TR} / \mathrm{TE}=6.6 \mathrm{~ms} / 1.9 \mathrm{~ms})$ in a $1.5 \mathrm{~T}$ magnet. Reconstruction slice thickness was $1.2 \mathrm{~mm}$, with an axial reconstruction matrix of $512 \times 512$ pixels spanning a $20 \mathrm{~cm}$ field-of-view. The time interval between pre-procedural imaging and intervention varied from days to weeks.

\subsection{General Segmentation Framework}

We used a model-based segmentation framework previously developed for CT 12 and MR [13. This approach appeared suitable for 3-D RA imaging since typical datasets show limited image quality and since an automatic, accurate and smooth representation of the LAPV anatomy is needed with minimal user interaction.

Shape-constrained Deformable Models. In the framework of shape-constrained deformable models, described in [12, mesh adaptation is performed by iterating two steps until the mesh reaches a steady state. First, boundaries are detected. Then, the mesh is deformed by minimizing the weighted sum of an external and an internal energy: $E=E_{\text {ext }}+\alpha E_{\text {int }}$. The external energy pulls the mesh to the detected boundaries. The internal energy penalizes deformations which deviate from a reference shape (e.g., mean shape) undergoing a geometric transformation (e.g., rigid or affine) to account for pose and shape variability.

Multi-stage segmentation. To increase robustness, we apply a multi-stage adaptation framework: The first step is the mesh initialization at a rough estimate of the cardiac center of mass based on the generalized Hough transform 
(GHT) [12 14 15. Second, the mesh is deformed parametrically, minimizing $E_{\text {ext }}$ alone. Specifically, pose misalignment is compensated by a similarity transform, followed by an affine transformation for non-isotropic scaling and possible shearing. Finally, a deformable adaptation is performed as described above (including $\left.E_{\text {int }}\right)$ to account for more local variations of the patient's anatomy [12].

Optimized Boundary Detection. Since vertex correspondence is preserved during mesh adaptation, we can assign a locally optimal boundary detection function to each mesh triangle using the Simulated Search approach 12 . Specifically, the magnitude of the image gradient (projected onto the triangle normal vector) together with several additional constraints on the gray values across the boundary are used for boundary detection. Suitable candidates for the constraints (e.g. gray values left or right of the boundary) and reasonable acceptance intervals for the constraints are estimated from the training images. The simulated search selects the optimal boundary detection function for each triangle from all candidates.

Image Intensity Calibration. To compensate for image intensity variations across images in 3-D RA and MRA data, we compute the image histogram and determine the low and high $2 \%$ percentiles 16 . The intensity values within the remaining histogram are then linearly re-scaled to a suitable reference interval.

\subsection{Pre-procedural CTA/MRA Segmentation}

For the pre-procedural CTA and MRA images, the entire heart was segmented to extract all four chambers, myocardium, and major vascular structures [17. The CT model (mean mesh, R-table for the GHT and optimal boundary detection functions) was trained on independent training data [17. The specific MR model for the MRA pulse sequence used was trained with a leave-5-out approach on the 15 pre-procedural MRA images (including image calibration, Sec. 2.3). After segmenting the whole heart in the pre-procedural volumes, the LAPV geometry was extracted by removing all triangles and vertices which did not correspond to the left atrium or pulmonary veins.

\subsection{3-D RA Reference Mesh Generation and Segmentation}

To train a 3-D RA LAPV model (mean mesh, R-table and optimal boundary detection function for each triangle), annotated reference meshes are required. We used a deforming mesh consisting of the endocardial surface of the LA and the surface of the four main PV trunks. This mesh is made of $V=1330$ vertices which form $T=2717$ triangles and has the same topology as that derived from the pre-procedural data (Fig. 3. left column). With this mesh topology, a reference mesh was generated manually for all 33 3-D RA data sets, matching the observable image boundaries as closely as possible. Unfortunately, the limited image quality leads to ambiguities in generating the reference meshes. In particular, the boundary between the left atrium (LA) and left ventricle (LV) is often not clearly visible and cannot easily be inferred from surrounding cardiac structures. To improve the delineation of the mitral annulus, we therefore reviewed 
our reference meshes in a second step based on registered LAPV meshes automatically segmented from the pre-procedural CTA or MRA data. This served to better define the mitral annulus in cases of ambiguous or missing image boundary in the 3-D RA data. The resulting reference meshes were again matched against the 3-D RA image data and, if necessary, manually refined.

Training and automatic segmentation of the 33 3-D RA data sets (Sec. 2.3) was performed using a leave-3-out approach.

\subsection{Registration of Pre-procedural and Intra-procedural Meshes}

In order to compare the automatic segmentations of pre-procedural and intraprocedural images, the resulting meshes were registered into a common coordinate system, based on a rigid registration transform whose parameters are estimated by minimizing the squared error measure $\sum_{i=1}^{V}\left|R \boldsymbol{v}_{i}^{\text {pre }}+\boldsymbol{t}-\boldsymbol{v}_{i}^{\text {intra }}\right|^{2}$. Here, $R$ is a $3 \times 3$ rotation matrix, $\boldsymbol{t}$ is a 3 -D translation vector, $\boldsymbol{v}_{i}^{\text {pre }}$ are the $V$ vertices of the automatically segmented pre-operative CTA or MRA mesh, $\boldsymbol{v}_{i}^{\text {intra }}$ are corresponding vertices of the automatically segmented 3-D RA mesh. Note that the registration parameters are optimized on both the pre-procedural meshes and the intra-procedural 3-D RA meshes. Independent parameter estimation e.g. based on intensity-based measures was not used due to the widely differing contrast characteristics encountered.

\subsection{Assessment of Segmentation Accuracy and Mesh Comparison}

The segmentation accuracy is assessed by measuring the Euclidean "surface-topatch" distance $\epsilon_{i, n}$ between the automatic segmentation of the 3-D RA data and a corresponding reference mesh (which is either the 3-D RA reference mesh, see Sec. 2.5, or the automatically segmented LAPV mesh of the pre-procedural data, Sec. 2.4). $\epsilon_{i, n}$ is defined as the distance between triangle center $i$ of the adapted 3-D RA mesh for patient $n$ to an anatomically corresponding patch of maximum geodesic radius $r=10 \mathrm{~mm}$ of the reference mesh and vice versa. Averaging over triangles $i$ gives the error $\epsilon_{n}$ per patient, and further averaging over the 33 patients gives the mean error $\epsilon_{\text {mean }}$. Note that the triangle positions near the artificial cut-planes which define the distal bounds of the truncated pulmonary veins are excluded from the error measurement (255 out of 2717 triangles), since the cut-planes do not relate to actual anatomy.

\section{$3 \quad$ Results}

Using the approach described in Sec. 2.3. a geometric LAPV model could be extracted fully automatically from all 33 rotational X-ray images within about 1 $30 \mathrm{~s}$. The average distance to the $3-\mathrm{D}$ RA reference meshes was $\epsilon_{\text {mean }}=1.3 \mathrm{~mm}$ $\left(\epsilon_{n}\right.$ ranged between 0.6 and $\left.2.1 \mathrm{~mm}\right)$, see Table 17 . This is slightly larger than the

\footnotetext{
${ }^{1}$ Algorithm not optimized for speed; workstation with Intel Xeon processors $(4 \times$ 2.8GHz) and 2GByte RAM.
} 
Table 1. Mean segmentation error $\epsilon_{\text {mean }}(\mathrm{mm})$ between the 3-D RA automatic and reference segmentations (a) and mean mesh distance $\epsilon_{\text {mean }}(\mathrm{mm})$ between the automatic 3-D RA and pre-procedural CTA / MRA meshes (b) for the left atrium and pulmonary veins $(\mathrm{PV})$, together with the error statistics of the triangle distances (of $T_{k}$ individual triangles per anatomic region $k$ in $N$ individual images, i.e. $T_{k} \times N$ data points).

a) 3-D RA automatic meshes versus 3-D RA reference meshes

\begin{tabular}{|c||c||c|c|c|c|}
\hline \multirow{2}{*}{ Anatomical region } & \multirow{2}{*}{$\epsilon_{\text {mean }}(\mathrm{mm})$} & \multicolumn{3}{|c|}{ Percentage of triangles with error range } \\
\cline { 3 - 6 } & & $<1.0 \mathrm{~mm}$ & $1.0-2.0 \mathrm{~mm}$ & $2.0-3.0 \mathrm{~mm}$ & $>3.0 \mathrm{~mm}$ \\
\hline \hline Whole LAPV mesh & 1.31 & 57.5 & 23.6 & 8.8 & 10.1 \\
\hline Left atrium & 1.50 & 53.4 & 23.0 & 9.8 & 13.8 \\
\hline Inf. left PV & 0.86 & 71.7 & 20.9 & 4.0 & 3.4 \\
\hline Inf. right PV & 0.92 & 65.6 & 25.7 & 6.2 & 2.5 \\
\hline Sup. left PV & 0.84 & 69.9 & 22.9 & 5.2 & 2.0 \\
\hline Sup. right PV & 1.25 & 51.4 & 30.3 & 12.1 & 6.2 \\
\hline
\end{tabular}

b) 3-D RA automatic meshes versus pre-procedural automatic meshes

\begin{tabular}{|c||c||c|c|c|c|}
\hline \multirow{2}{*}{ Anatomical region } & \multirow{2}{*}{$\epsilon_{\text {mean }}(\mathrm{mm})$} & \multicolumn{3}{|c|}{ Percentage of triangles with error range } \\
\cline { 3 - 6 } & & $<1.0 \mathrm{~mm}$ & $1.0-2.0 \mathrm{~mm}$ & $2.0-3.0 \mathrm{~mm}$ & $>3.0 \mathrm{~mm}$ \\
\hline \hline Whole LAPV mesh & 2.27 & 29.2 & 27.2 & 17.8 & 25.7 \\
\hline Left atrium & 2.19 & 30.3 & 26.2 & 17.5 & 26.1 \\
\hline Inf. left PV & 1.64 & 39.6 & 31.1 & 15.0 & 14.4 \\
\hline Inf. right PV & 2.20 & 26.4 & 31.0 & 19.1 & 23.5 \\
\hline Sup. left PV & 2.01 & 26.9 & 32.4 & 20.9 & 19.9 \\
\hline Sup. right PV & 3.43 & 19.4 & 23.1 & 18.4 & 39.1 \\
\hline
\end{tabular}

distances reported for segmentation of CTA and MRA data 12 13. This finding can likely be attributed to the lower image quality of 3-D RA data compared to CTA and MRA. Low segmentation errors were observed particularly within the posterior aspect of the left atrium as well as at the proximal trunks of the pulmonary veins (Fig. 2a). These anatomical features are strongly constrained by pericardial tissue and are therefore not affected as much by motion artifacts in reconstruction. As a result, the boundaries of these areas are more sharply defined than the borders located closer to the ventricular surfaces which suffer from significant motion blurring. This motion artifact and concomitant lack of boundary contrast explains the difficulty of segmentation at the anterior aspect of the left atrium, particularly in regions close to the mitral annulus (Fig. $2 \mathrm{~b}$ ).

On the other hand, the average segmentation error is still significantly smaller than the average mesh distance of the 3-D RA meshes to the pre-procedural CTA or MRA LAPV meshes $\epsilon_{\text {mean }}=2.3 \mathrm{~mm}\left(\epsilon_{n}\right.$ ranged between 1.4 and $\left.4.9 \mathrm{~mm}\right)$, see Table 1 1b. A smaller or comparable segmentation error $\epsilon_{n}$ of the 3-D RA mesh as compared to its distance to the pre-procedural mesh was obtained for each patient individually. In particular, the posterior aspect of the left atrium and the proximal trunks of the pulmonary veins show somewhat large deviations between the automatically segmented 3-D RA mesh and the pre-procedural mesh (Fig. 2 c,d). Some examples for the mesh comparison are presented in Fig. 3 . 
1.) autom. 3-D RA vs. ref. 3-D RA

a) posterior view

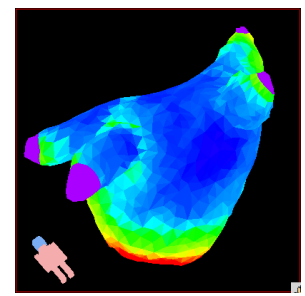

b) anterior view

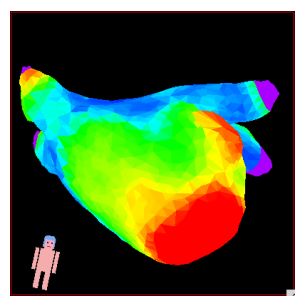

2.) autom. 3-D RA vs. autom. pre-proc.

c) posterior view

d) anterior view

Fig. 2. Color-code representation of mean mesh distances $\epsilon_{i}$ per triangle (averaged over all 33 patients) of the automatically segmented intra-procedural 3-D RA meshes to the 3-D RA reference meshes (a: posterior view, b: anterior view) and to the automatic pre-procedural CTA / MRA meshes (c: posterior view, d: anterior view). Dark blue: 0mm, dark red: $2.8 \mathrm{~mm}$, violett: triangles excluded from error measurement. The mesh distances are displayed on the mean 3-D RA reference mesh. The mitral valve (bottom part) does not correspond to an observable image boundary and thus has a large error.

Mean LAPV mesh preop. data (for 3-D RA)
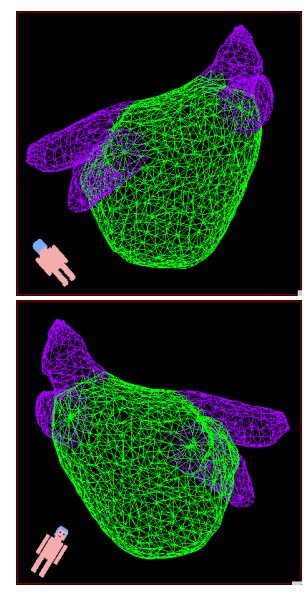

preop. mesh

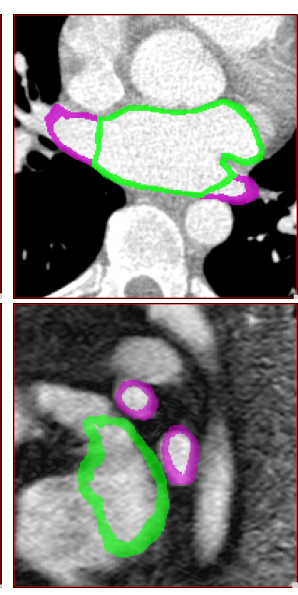

3-D RA data

3-D RA mesh

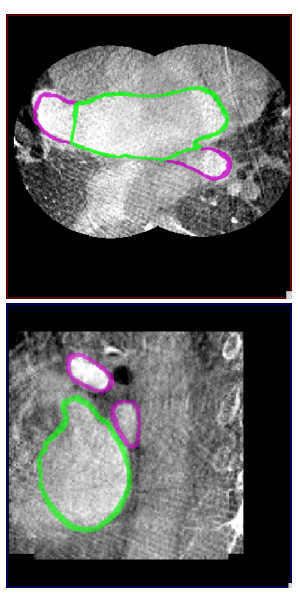

3-D RA data

preop. mesh

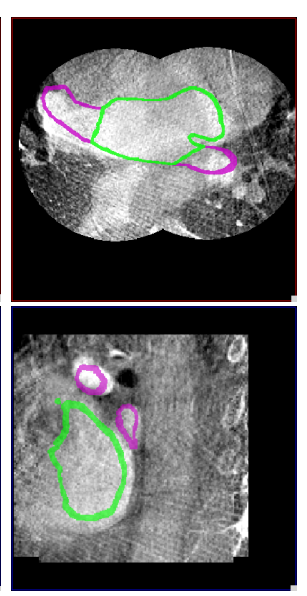

Fig. 3. Mean 3-D RA LAPV mesh from two different views (first column), and two typical examples for comparison of automatic LAPV segmentations in different modalities. Second column: cut-out of pre-procedural CTA (top) / MRA (bottom, different scaling factor) and corresponding automatic mesh, third column: intra-procedural 3-D RA data and corresponding automatic mesh, fourth column: 3-D RA data with registered CTA / MRA mesh. First row: axial view of patient 10 (deviation between 3-D RA and CTA mesh: $\epsilon_{n}=2.6 \mathrm{~mm}$ ), second row: sagittal view of patient 15 (deviation between 3-D RA and MRA mesh: $\epsilon_{n}=1.9 \mathrm{~mm}$ ). 


\section{Discussion and Conclusion}

To the best of our knowledge, we have developed and presented the first approach to fully-automatic and robust generation of left atrium and pulmonary vein models from rotational X-ray angiography (3-D RA). Despite the challenges faced in this segmentation task due to limited image quality and contrast available, geometric LAPV models could be reliably extracted from the intra-procedural 3-D RA data in a fully-automated fashion for all 33 patients. The resulting models can be used within the existing interventional EP workflow for improved catheter navigation and ablation guidance. The 3-D RA segmentation error for each patient was either comparable to or smaller than the mesh deviation between the intra-procedural 3-D RA mesh and a corresponding pre-procedurally acquired CTA or MRA mesh. These differences can be caused by several error sources: (1) Anatomical or physiological variation between the pre-procedural and the intraprocedural image acquisition, (2) 3-D RA image acquisition artifacts, e.g. due to heart motion (no ECG gating, in contrast to CTA), (3) segmentation inaccuracies of 3-D RA and/or pre-procedural data. Currently, our ability to discriminate between these error sources is limited, primarily due to limitations of image acquisition and lack of an unambiguous ground truth for the 3-D RA data. With this in mind, our results indicate that intra-procedural LAPV models from 3-D RA are accurate and comparable to models derived from pre-procedural CTA or MRA data, with the additional advantage of representing the cardiac anatomy at the time of the EP intervention itself. Future work includes the handling of a broader variety of anatomical PV variants that occur less frequently than those observed in our study population.

Acknowledgments. Authors would like to thank Drs. A. d'Avila, A. Thiagalingam, A. H. Locke and Z. Malchano for help with the data acquisition.

\section{References}

1. Haissaguerre, M., Jais, P., Shah, D.C., et al.: Spontaneous initiation of atrial fibrillation by ectopic beats originating in the pulmonary veins. New Engl. J. Med. 339, 659-666 (1998)

2. Pappone, C., Oreto, G., Rosanio, S., et al.: Atrial electroanatomic remodeling after circumferential radiofrequency pulmonary vein ablation: efficacy of an anatomic approach in a large cohort of patients with atrial fibrilation. Circulation 104, 25392544 (2001)

3. Rhode, K., Hedge, S., Sermesant, M., et al.: XMR guided cardiac electrophysiology study and radio frequency ablation. In: Proc. SPIE Med. Imag. (2004)

4. Reddy, V.Y., Malchano, Z.J., Holmvang, G., et al.: Integration of cardiac magnetic resonance imaging with three-dimensional electroanatomic mapping to guide left ventricular catheter manipulation. Journal of the American College of Cardiology 44(1), 2202-2213 (2004)

5. Nollo, G., Cristoforetti, A., Faes, L., et al.: Registration and fusion of segmented left atrium CT images with CARTO electrical maps for the ablative treatment of atrial fibrillation. Computers in Cardiology, 345-348 (2004) 
6. von Berg, J., Lorenz, C.: Accurate left atrium segmentation in multislice CT images using a shape model. In: Proc. SPIE Med. Imag., vol. 5747, pp. 351-360 (2005)

7. John, M., Rahn, N.: Automatic left atrium segmentation by cutting the blood pool at narrowings. In: Duncan, J.S., Gerig, G. (eds.) MICCAI 2005. LNCS, vol. 3750, pp. 798-805. Springer, Heidelberg (2005)

8. Karim, R., Mohiaddin, R., Rueckert, D.: Left atrium segmentation for atrial fibrillation ablation. In: Proc. SPIE Med. Imag. (in press, 2008)

9. Thiagalingam, A., Manzke, R., D'Avila, A., et al.: Intraprocedural volume imaging of the left atrium and pulmonary veins with rotational X-ray angiography: implications for catheter ablation of atrial fibrillation. J. Cardiovasc. Electrophysiol. 19, 293-300 (2008)

10. Manzke, R., Reddy, V.Y., Dalal, S., et al.: Intra-operative volume imaging of the left atrium and pulmonary veins with rotational x-ray angiography. In: Larsen, R., Nielsen, M., Sporring, J. (eds.) MICCAI 2006. LNCS, vol. 4190, pp. 604-611. Springer, Heidelberg (2006)

11. Lauritsch, G., Boese, J., Wigström, L., et al.: Towards cardiac C-arm computed tomography. IEEE Trans. Med. Imaging 25, 922-934 (2006)

12. Ecabert, O., Peters, J., Schramm, H., et al.: Automatic model-based segmentation of the heart in CT images. IEEE Trans. Med. Imaging (in press, 2008)

13. Peters, J., Ecabert, O., Meyer, C., et al.: Automatic whole heart segmentation in static magnetic resonance image volumes. In: Ayache, N., Ourselin, S., Maeder, A. (eds.) MICCAI 2007, Part II. LNCS, vol. 4792, pp. 402-410. Springer, Heidelberg (2007)

14. Ballard, D.H.: Generalizing the Hough transform to detect arbitrary shapes. Pattern Recogn. 13(2), 111-122 (1981)

15. Brejl, M., Sonka, M.: Object localization and border detection criteria design in edge-based image segmentation: Automated learning from examples. IEEE Trans. Med. Imag. 19(10), 973-985 (2000)

16. Nyul, L.G., Udupa, J.K.: On standardizing the MR image intensity scale. Magnetic Resonance in Medicine 42, 1072-1081 (1999)

17. Peters, J., Ecabert, O., Lorenz, C., et al.: Segmentation of the heart and major vascular structures in cardiovascular CT images. In: Proc. SPIE Med. Imag. (in press, 2008) 